\title{
AN ELEMENTARY PROOF THAT ALL SPANNING SURFACES OF A LINK ARE TUBE-EQUIVALENT
}

\author{
DROR BAR-NATAN, JASON FULMAN, AND LOUIS H. KAUFFMAN
}

\begin{abstract}
The standard proof that the potential function models the Alexander-Conway polynomial depends on the fact that all spanning surfaces of a link are tube-equivalent [K1]. Proofs that all spanning surfaces of a link are tube-equivalent use machinery such as the Thom-Pontrjagin construction [L]. Here we present an elementary geometric argument in the case of links in $S^{3}$ which allows one to visualize the additions and removals of tubes.
\end{abstract}

\section{CONTENTs}

1. Introduction 1

2. Some background and preliminary work 2

3. Proof of Theorem $1 \quad 4$

References $\quad 7$

\section{INTRODUCTION}

Let $L$ be an oriented 1-dimensional link in $S^{3}$. A spanning surface for $L$ is a compact, oriented two-dimensional manifold $S$ in $S^{3}$ such that $S$ has oriented boundary $L$ (for technical convenience, we've dropped the connectivity assumption. This only makes our results below more general). Two spanning surfaces are called tube-equivalent or S-equivalent if they are related up to ambient isotopy by the addition or removal of tubes, where each addition of a tube is required to preserve the orientability of the surface. See for instance Figure 1.

Figure 1. Two parts of a spanning surface, before and after the addition of a tube.
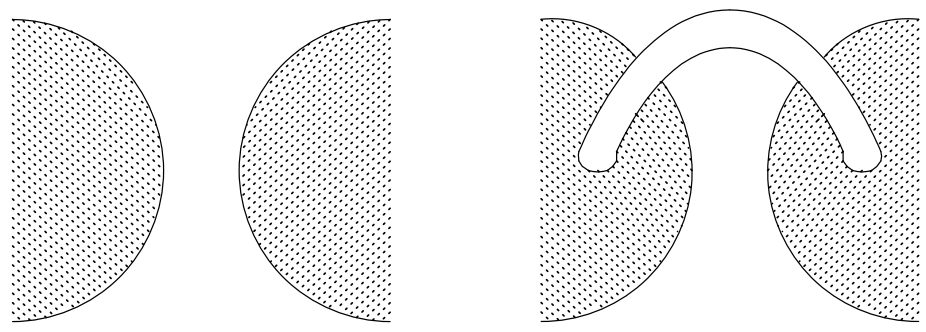

Our main theorem is:

Theorem 1. Let $S_{1}$ and $S_{2}$ be two spanning surfaces for the same link L. Then $S_{1}$ and $S_{2}$ are tube-equivalent.

Date: This edition: March 28, 1998; First edition: June 20, 1995.

This work was supported by NSF grant DMS-92-03382.

This preprint is available electronically at http://www.ma.huji.ac.il/ ${ }^{d r o r b n}$ and at http://www.dartmouth. edu/ jfulman/index.html. 
This theorem is well known; see e.g. [K1]. The theorem appears to be the only nonelementary part in the proof (appearing there) that the potential function provides a model for the Alexander-Conway polynomial, and its standard proof uses some facts about Morse theory and 3-dimensional manifolds. The purpose of this note is to give an elementary proof of Theorem 1, using only standard knot-theoretic arguments.

\section{SOME BACKGROUND AND PRELIMINARY WORK}

A surface is said to be in band-handle form if, as in Figure 2, it is a standardly embedded disk with bands attached (these bands may be twisted or linked). It follows from the classification theorem for 2 dimensional compact manifolds that every spanning surface is abstractly homeomorphic to a surface in band-handle form. By shrinking a spanning surface towards its 1-dimensional skeleton one concludes that every spanning surface is ambient isotopic to a surface in band-handle form. This is discussed in [K2, pp. 81].

Figure 2. A surface in band-handle form.

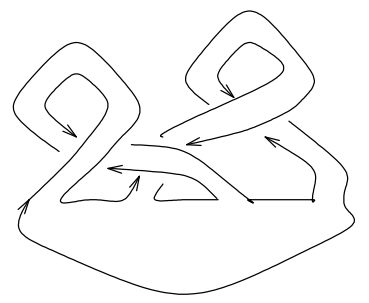

There is an algorithm, due to Seifert [S], which constructs a spanning surface for any oriented link $L$, given a planar projection of that link. As our proof makes use of this algorithm, we describe it as follows. For more details see [K2]. A surface produced by Seifert's algorithm is called a Seifert surface or an algorithm surface.

1. At each crossing in the projection draw arcs as in Figure 3. Then form a set of disjoint closed curves, called Seifert circles, by tracing along the edges of the projection and moving along the arcs when arriving at a crossing. (These circles may be nested).
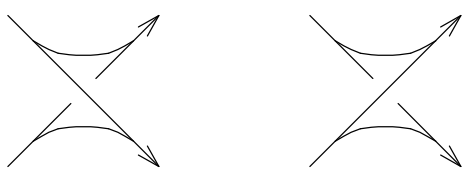

Figure 3. Adding arcs near crossings in a link projection.

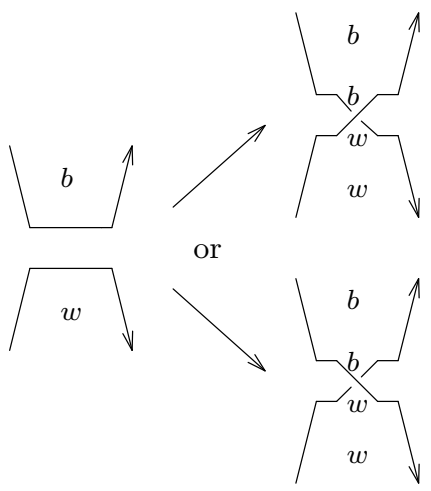

Figure 4. Addings bands between Seifert circles.

2. Define the depth $d(C)$ of a Seifert circle $C$ to be the number of Seifert circles in which it is properly contained. Translate each circle $C$ upward (toward the reader) by $d(C)$ units, and attach a disk inside each Seifert circle. Assign "colors" (say black $b$ and 
white $w$ ) to the sides of each disk by the convention that the oriented boundary runs counterclockwise as seen from the black $(b)$ side.

3. Between Seifert circles where a crossing existed, add twisted bands, according to the crossing in $L$, as in Figure 4. Note that this is always possible, since if a crossing existed between Seifert circles, their depths differed by at most 1. It is easy to see that the coloring of the disks can be continued across the twisted bands, and thus the resulting surface is orientable.

Figure 5 shows the result of Seifert's algorithm applied to a projection of the trefoil knot.

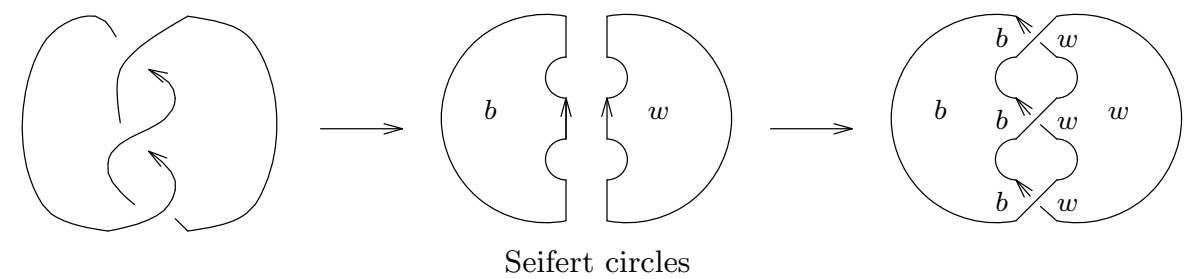

Figure 5. A Seifert surface for the trefoil knot.

Remark 2.1. One can easily check that the result of the above algorithm depends only on the projection of $L$ considered as a graph (with crossing information) in $S^{2}$. In other words, the resulting surface does not change when curves in the projection are moved "across infinity".

In the proof of Theorem 1, we will use the familiar three Reidemeister moves, connecting any planar projection of $L$ to any other such projection $[\mathrm{R}]$ :

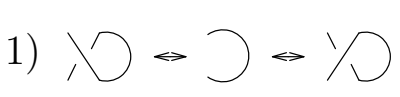

2)

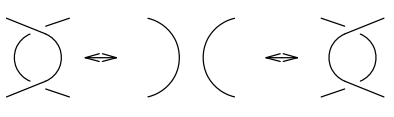

3)

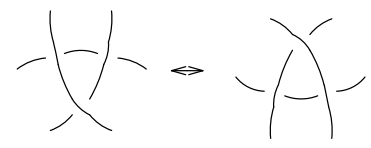

It is helpful to introduce some notation. The symbols + and - are for crossings as in Figure 6. The symbol ? means that the crossing can be either + or - . The symbol $\mid$ denotes a Seifert circle, and the symbols,,$+- \mid$ can be combined as in Figure 7. Note that the arrows indicate orientation, but need not be included if the orientation chosen is irrelevant. Distinct I's correspond to distinct Seifert circles. The relative heights of the circles involved are not relevant, and hence are not indicated pictorially. The symbol $|T|$ will denote a tube as in Figure 1.

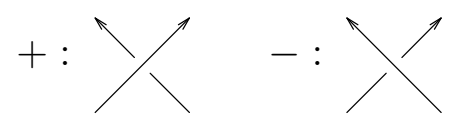

Figure 6. Two types of crossings.
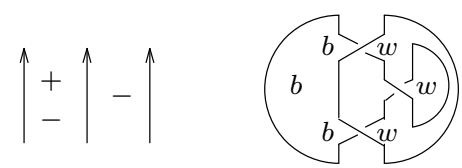

Figure 7. Example of notation.

Lemma 2.2. Connecting two surfaces with two adjacent twisted bands with opposite twists is equivalent to adding a tube between them. Symbolically, the relation $\quad| \pm|=|T|=|\mp|$ holds. 
Proof. The proof is clear from the figure on the right, which shows the case where the two surfaces are disks. The disks can be pulled apart, and the region with lined arrows becomes the boundary of the tube between them. A similar argument shows that the theorem is true if we switch the + and - .

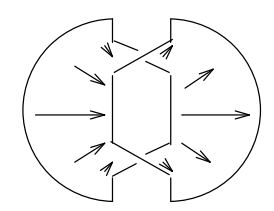

Lemma 2.3. The following four relations hold:
1
$2 . \uparrow+\uparrow+\uparrow \sim \uparrow+\uparrow+\uparrow$
3. $\stackrel{?}{?}-\uparrow-\uparrow \sim \uparrow-\uparrow-\uparrow ?$
4. $\uparrow-\uparrow \begin{gathered}? \\ -\end{gathered} \sim \uparrow-\uparrow ?-\uparrow$

That is, surfaces with local crossing configurations as indicated above are tube-equivalent.

Remark 2.4. Note that the cases 1-4 in Lemma 2.3 enumerate all of the type 3 Reidemeister moves in braided form, with all lines oriented in the same braiding direction.

Proof of Lemma 2.3. To prove the first relation, slide the left and right sides of ? down along the two thickened lines in the picture shown on the right, until it becomes a twisted band between $A$ and $B$, instead of between $B$ and $C$. The second relation in the case of $?=+$ is the same as the first relation in the case of $?=+$, so it also follows.

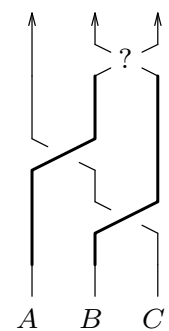

The second relation in the case of $?=-$ is only a bit more difficult, and the proof in that case uses the first relation as well as two applications of Lemma 2.2:

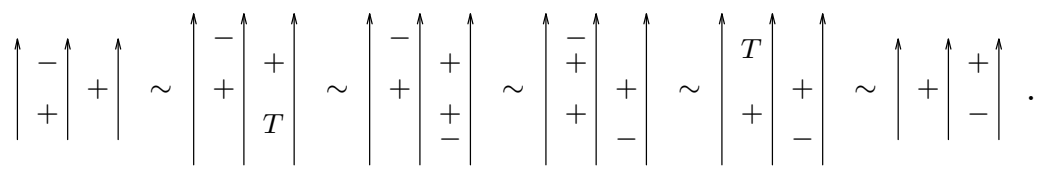

The third and fourth relations are proven in a similar way.

\section{Proof of Theorem 1}

As stated in Section 2, every spanning surface is ambient isotopic to a surface in bandhandle form. We may thus assume $S_{1}$ and $S_{2}$ are in band-handle form.

The proof has two steps. The first step is to show that a spanning surface in band-handle form is tube-equivalent to an algorithm surface. The second step is to fix link projections $L_{1}$ and $L_{2}$ of $L$ and show that the algorithm surfaces of $L_{1}$ and $L_{2}$ are tube-equivalent. This will be done by analyzing how performing a Reidemeister move on a link projection affects the corresponding algorithm surface. This second step is inspired in part by a remark in [K2, pp. 198].

For the first step of the proof, suppose the spanning surface is in band-handle form. We want to show that it is tube equivalent to the algorithm surface. As is shown in Figure 8, adding a local twist to the surface in band-handle form does not change the algorithm surface. Thus we may assume (by performing a local twist on one of the bands if necessary), that at every crossing in the band-handle surface, the two bands are oriented as in Figure 9. Having the bands oriented as in Figure 9 guarantees that the Seifert circles from the algorithm 
surface lie inside the two bands (as opposed to lying in the complement of the bands). In fact, as is illustrated in Figure 10, the algorithm surface can be obtained from the surface in band-handle form by adding a tube between the lower band and upper band. Since the tube is added between two black sides, the surface so obtained is orientable. The regions marked 1 and 2 in Figure 10 correspond to the lower band and upper band respectively. The vertical tube is also indicated. The arrows on the boundary of the tube point in the direction of increasing height.

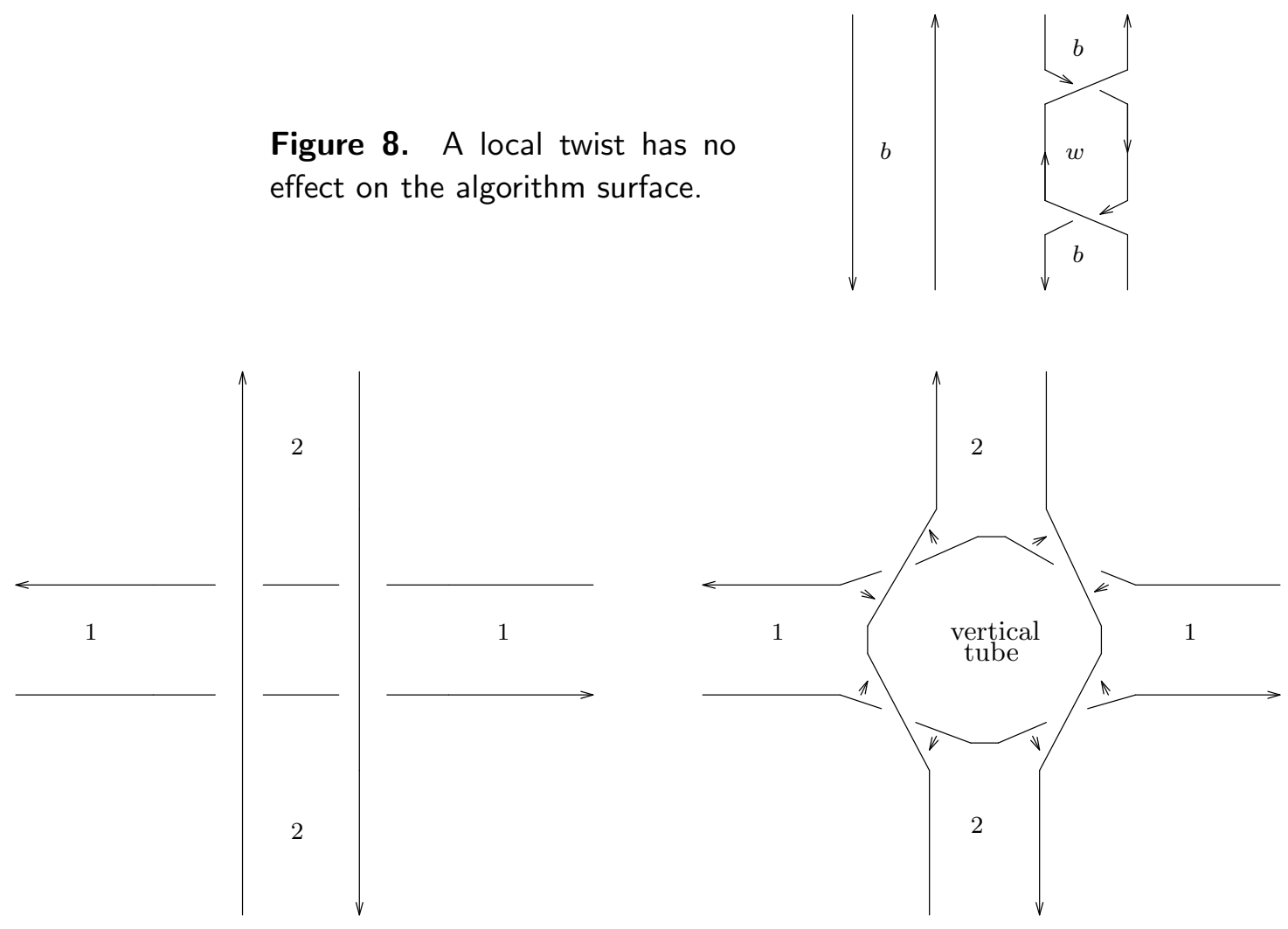

Figure 9. A crossing in the spanning surface.

Figure 10. Tube equivalence of band-handle surface and algorithm surface.

We now proceed to the second step of the proof by analyzing how the algorithm surface changes under each of the Reidemeister moves.

For the first Reidemeister move, there is essentially only one case to consider and it is shown in Figure 11. As is also clear from Figure 11, the first Reidemeister move leaves unchanged the surface produced by Seifert's algorithm.

Figure 11. The effect of Move 1 on the algorithm surface:

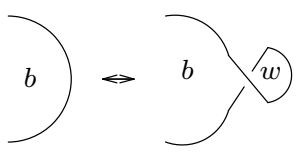

For the second Reidemeister move, assume that the circle on the left moves over the circle on the right. There are three cases to analyze (see Figure 12) depending on the orientations of the strands involved and on the way they close into Seifert circles. (Some more possibilities 
are reduced to these three by using Remark 2.1). Figure 12 shows the algorithm surface before and after the second Reidemeister move is performed. One easily sees the tube-equivalence of the 'before' and 'after' surfaces in each case: In case 1, just untwist the middle " $w$ " in the 'after' surface. In case 2, use Lemma 2.2. In case 3, untwist the middle $w$ in the 'after' surface. The result is easily seen to be the same as the 'before' surface, with one tube added as in Figure 1 between the two $w$ disks.
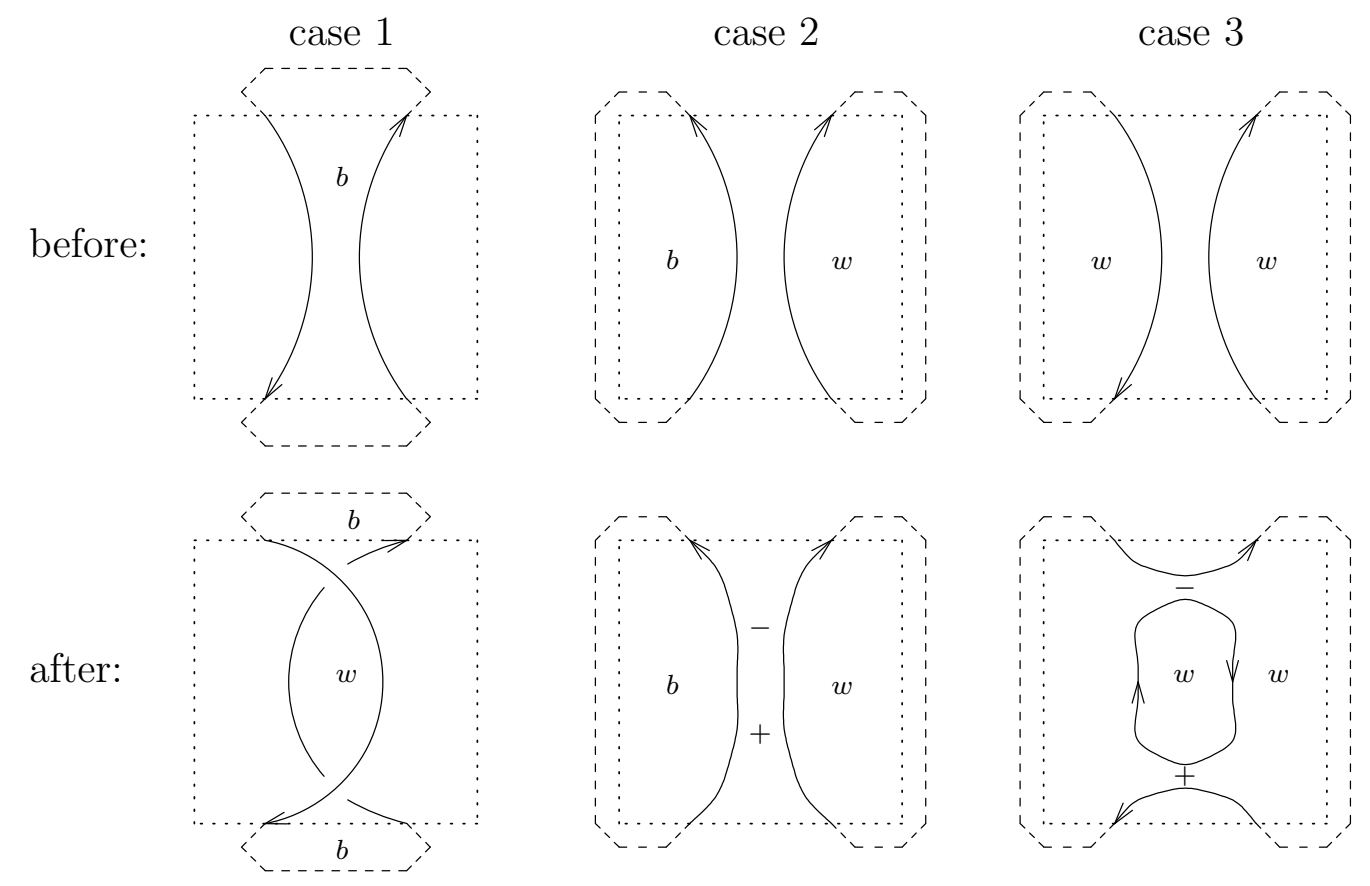

Figure 12. The three cases of the second Reidemeister move.

For the third Reidemeister move, there are four cases based on the orientations of the three strands involved (see Figure 13). The dotted boundary which appears in all figures involving the third move represents a small sphere in which all ambient isotopies and additions and removals of tubes will occur. This emphasizes that all arguments which follow are local.
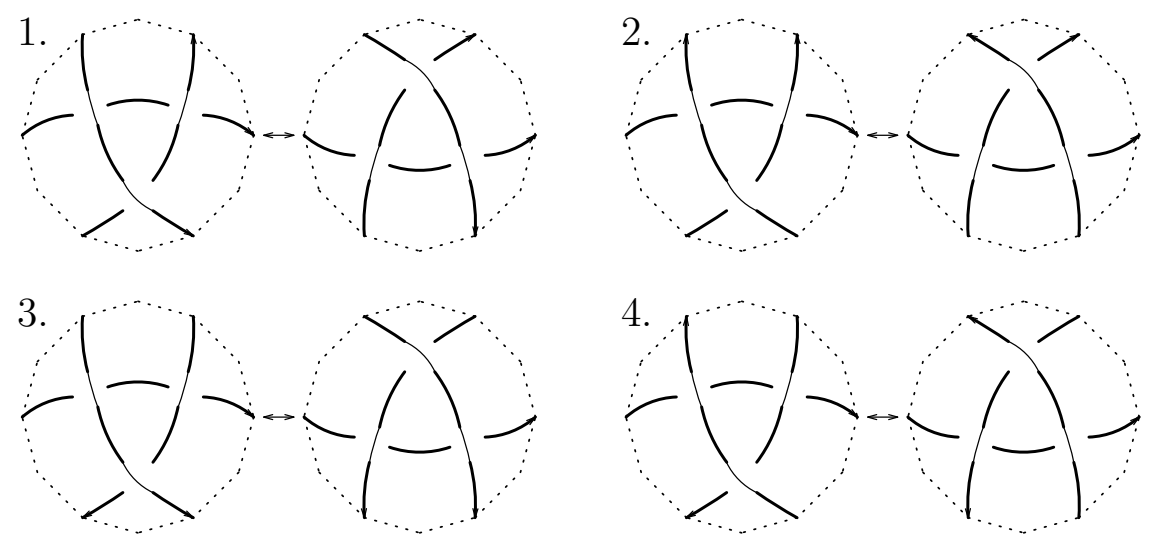

Figure 13. Possible cases of Move 3. 
Cases 1-3 follow from Lemma 2.3. This is indicated in Figure 14. Case 4 differs from cases 1-3 by Reidemeister moves of type 2, as shown in Figure 15. Hence the algorithm surfaces for the two sides of case 4 are tube-equivalent by the work we have already done.

Figure 14. The effects of cases 1-3

1.

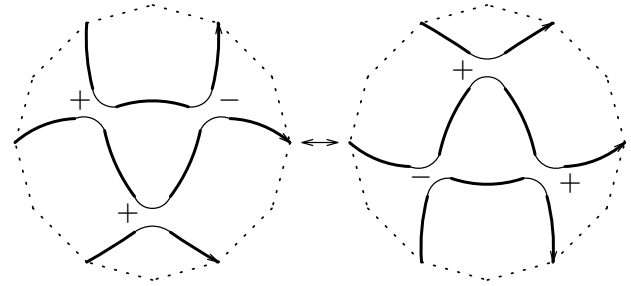

3.

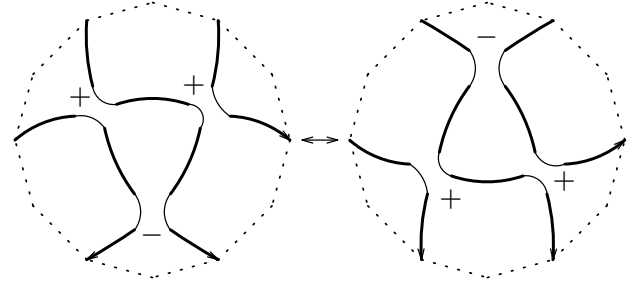

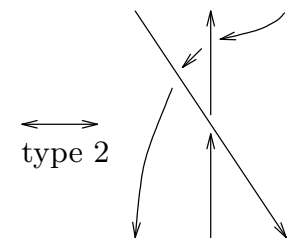

Figure 15. A case 4 move is a composition of four type 2 moves and a single move from cases 1-3.

\section{REFERENCES}

[K1] L. H. Kauffman, The Conway polynomial, Topology 20 (1980) 101-108.

[K2] _ On knots, Princeton Univ. Press, Princeton 1987.

[L] J. Levine, Knot cobordism groups in codimension two, Comm. Math. Helv. 44 (1969) 229-244.

[R] K. Reidemeister, Knotentheorie, Chelsea, New-York 1948 (Copyright Julius Springer, Berlin 1932).

[S] H. Seifert, Uber des geschlect von knoten, Math. Annals 110 (1934) 571-592.

Department of Mathematics, Harvard University, Cambridge, Massachusetts 02138, USA Current address: Institute of Mathematics, The Hebrew University, Giv'at-Ram, Jerusalem 91904, Israel E-mail address: dror@math.huji.ac.il

Department of Mathematics, Harvard University, Cambridge, Massachusetts 02138, USA Current address: Department of Mathematics, 6188 Bradley Hall, Dartmouth College, Hanover, New Hampshire 03755, USA

E-mail address: fulman@dartmouth.edu

Department of Mathematics, University of Illinois at Chicago, 851 South Morgan Street, Chicago, Illinois 60607-7045, USA

E-mail address: kauffman@uic.edu 